\title{
An Epsilon-Near-Zero (ENZ) Based, Ultra-Wide Bandwidth Terahertz Single-Polarization Single-Mode Photonic Crystal Fiber
}

\author{
Tianyu Yang, Can Ding, Member, IEEE, Richard W. Ziolkowski, Life Fellow, IEEE and Y. Jay Guo, Fellow, IEEE,
}

\begin{abstract}
A novel terahertz (THz) photonic crystal fiber (PCF) that yields single-polarization single-mode (SPSM) propagation over an ultra-wide bandwidth is designed and analyzed. The PCF is based upon a triangle-based lattice of air holes in a high resistivity silicon substrate with three selectively-filled rectangular slots introduced into the core area. Four air holes surrounding the core region are chosen to be loaded with an epsilon-near-zero (ENZ) material. The configuration and the large loss of the ENZ material establish a large loss difference (LD) between the two fundamental propagating polarization modes and any higher order modes. When the central slot of the three in the core is filled with a gain material and the adjacent two slots are air-filled, the $L D$ values between the one desired propagating mode and all other modes are significantly enhanced. Consequently, essentially only the desired mode will exist in the PCF after a short propagation distance resulting in the SPSM behavior. The optimized design provides large $L D$ values, greater than $9.4 \mathrm{~dB} / \mathrm{cm}$, over a SPSM spectrum of $0.64 \mathrm{THz}$ (from 1.10 to $1.74 \mathrm{THz}$ ), which, to the best of our knowledge, is the widest SPSM bandwidth achieved to date in the $\mathrm{THz}$ regime. The unwanted modes are $30 \mathrm{~dB}$ smaller than the wanted mode after a $3.2 \mathrm{~cm}$ length of the PCF. This outcome is highly desired for polarization sensitive $\mathrm{THz}$ communications and sensor systems that rely on waveguiding structures.
\end{abstract}

Index Terms-terahertz, fiber characteristic, singlepolarization singel-mode.

\section{INTRODUCTION}

$\mathbf{T}$ $\mathrm{O}$ meet the ever increasing capacity requirements of data transfers, terahertz $(\mathrm{THz})$ band systems are expected to be the next frontier of wireless communications [1]. The $\mathrm{THz}$ band, which is generally defined in the range from $0.1-10 \mathrm{THz}$ (30-3000 $\mu \mathrm{m})$, has attracted significant world-wide attention in the last decade. This interest arises from the availability of wide bands of unallocated spectrum that could be used for various sensing, imaging, security, and communication applications [2]. Among the current waveguiding structures that support wireless applications in the $\mathrm{THz}$ range, e.g., coplanar transmission lines, metallic waveguides, and dielectric waveguides, fiber-based waveguiding systems are the most promising candidates due to their low transmission losses and controllable guiding characteristics [2].

Wave guiding that is polarization maintaining (PM) is a preferred or often requested characteristic in both $\mathrm{THz}$ and optical

This work was supported by Australian Research Council (ARC) DECRA grant number DE200101347.

T. Yang, C. Ding, R. W. Ziolkowski, and Y. J. Guo are with the Global Big Data Technologies Centre, University of Technology Sydney, Ultimo, NSW 2007, Australia.

Corresponding author: Can Ding, can.ding.1989@gmail.com. fiber applications. This feature overcomes the crosstalk and modal dispersion between the two fundamental, orthogonal polarization, propagating modes in a variety of communication systems. PM fibers were first invented by forcing those two modes to propagate with different phase velocities to the benefit of sensitive communication systems and sensors [3], [4]. Typical ones include PANDA (polarization-maintaining and absorption-reducing) and elliptical-cladding fibers [4], [5]. However, their PM abilities are limited due to their low birefringence values $\left(10^{-4}\right)$.

On the other hand, photonic crystal fibers (PCFs) have attracted considerable attention in the last two decades as a consequence of their outstanding advantages, e.g., tunable dispersion, endless single mode, large nonlinearities, and design flexibilities [6]. Moreover, PCFs can achieve higher birefringence values, i.e., from $10^{-2}$ to $10^{-1}$. To date, a variety of $\mathrm{THz}$ and optical highly birefringent PCFs (HB PCFs) have been investigated both theoretically and experimentally [713]. Their enhanced PM performance has been facilitated by introducing asymmetries into their configurations. For instance, a circular-hole based HB PCF achieved a high birefringence value, 0.26 , at $1.55 \mu \mathrm{m}$ with the introduction of an asymmetrical arrangement of its holes in both its core and cladding [7]. A fabricated rectangular HB PCF with a porous core obtained a birefringence value of 0.012 at $0.65 \mathrm{THz}$ [8]. A dual-hole unit-based porous-core PCF yielded a birefringence value of 0.033 at $0.85 \mathrm{THz}$ [9]. Higher birefringence values have been demonstrated with hybrid and complicated designs, e.g., a epsilon-near-zero (ENZ) material filled PCF attained a birefringence over 0.1 at $1 \mathrm{THz}$ [10]. However, the introduction of birefringence in PCFs only reduces the crosstalk and modal dispersion; it does not eliminate those issues.

An alternative solution to reduce crosstalk is to use another type of PM PCF called a single-polarization-single-mode PCF (SPSM PCF). It is designed to only support one specific propagating polarization mode. SPSM PCFs have been successfully deployed in the optical regime as a consequence of the maturity of the optical fiber technologies. Reported SPSM PCFs are mainly based on two methods. One is the index matching coupling technique [14, 15], which employs the introduction of index-matched cladding defects to make the attenuation rates of the two fundamental modes different. The second is the cut off method [16, 17], which is based on varying the dimensions and/or shapes of the air holes and lattice constant of the PCF design to achieve different cutoff frequencies of the two fundamental modes. Despite the 
increasing importance of $\mathrm{THz}$ communications, few works on THz SPSM PCFs have been reported. While various optical SPSM PCFs have been realized, the larger losses in the $\mathrm{THz}$ regime have hindered similar developments.

The total loss (TL) of PCFs is generally composed of the confinement loss (CL) and the effective material absorption loss (EML). The CL is the dominant factor for optical PCFs. On the other hand, the EML is the dominant component for THz PCFs. The aforementioned two SPSM approaches focus on adjusting the geometrical configurations to introduce asymmetries to the electric field (E-field) distributions within the PCF. Thus, they both introduce a loss difference (LD) between the CLs of the two fundamental modes. While both approaches work for optical PCFs, the generally obtained LD values in $\mathrm{THz}$ PCFs are not large enough to attain SPSM operation. For example, the minimum loss difference (MLD) between the wanted mode and all other unwanted modes of the reported $\mathrm{THz}$ PCFs based on the index matching coupling technique [15] and the cut off method [17] are only 0.036 $\mathrm{dB} / \mathrm{cm}$ and $0.06 \mathrm{~dB} / \mathrm{cm}$, respectively. Consequently, one must focus on the EML values in the THz PCFs to achieve larger $\mathrm{LD}$ values between the wanted and unwanted modes. A THz SMSP PCF has been designed by depositing ENZ material in the cladding and doping gain material in the core to manipulate the EML values of different modes [18]. The MLD between the wanted and all unwanted modes was $>8.0 \mathrm{~dB} / \mathrm{cm}$ across the operational SPSM band, $0.312 \mathrm{THz}$. While the MLD values were much larger than those obtained by manipulating the $\mathrm{CL}$ values, the reported configuration was rather simplistic, and it was found that that its performance characteristics could be significantly improved with an innovative redesign.

An advanced THz SPSM PCF inspired by our previous work [18] is developed in this paper. Fine control of the E-field distributions of both fundamental and all higher order modes is achieved by introducing appropriately dimensioned rectangular slots into the core region and strategically repositioning the ENZ ring-loaded air holes in the first ring of the cladding region. While the overall operating principles of the design are more sophisticated, they are not intrinsically more complex. The combination of rectangular slots and repositioned ENZ rings leads to fine control of the E-field distribution of both fundamental and all higher order modes. By regulating the differences between the E-field distributions of the wanted and unwanted modes, the overlap of the unwanted modes with the lossy ENZ rings is enhanced and leads to superior SPSM performance characteristics of the wanted mode. With a final optimization of the orientation and positions of the ENZ rings relative to the slots and of the dimensions of the air-filled and gain-impregnated slots, an even wider bandwidth, $0.64 \mathrm{THz}$, is achieved along with an improved MLD value, $>9.4 \mathrm{~dB} / \mathrm{cm}$. Compared to our previous work [18], the SPSM bandwidth of this slightly more complex configuration is $106 \%$ wider and the MLD value is improved by $17.5 \%$. Finally, an overall comparison between the developed designs and reported stateof-the-art works will be presented to emphasize their superior performance characteristics.

All of the simulations involved in this work were conducted with the full-wave vector finite element method (FEM) based

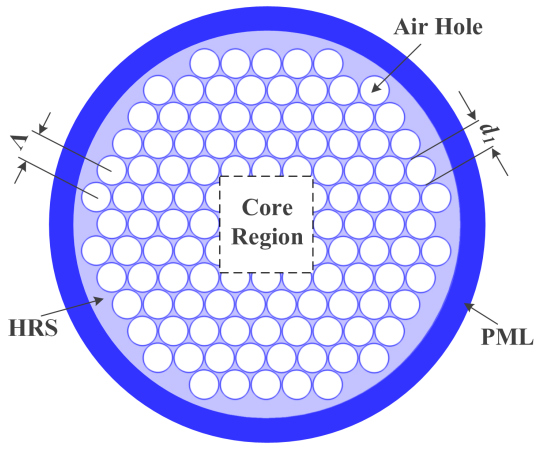

(a)

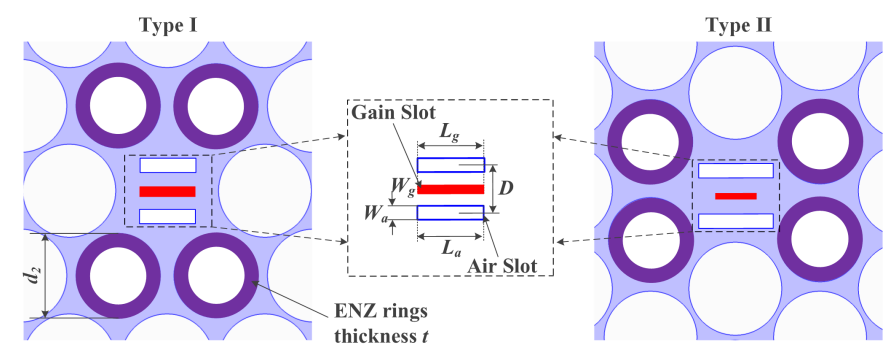

(b)

Fig. 1. Cross sectional views of the slotted SPSM PCFs.

COMSOL Multiphysics commercial software package [19]. A perfectly match layer (PML) whose thickness is $10 \%$ of the whole diameter of the PCF was set concentrically on the outside of the cladding to properly truncate the numerical simulation space. Its eigen-mode solver was adopted to calculate the modes with the convergence value set to $10^{-6}$. With this convergence value and the software's adaptive meshing, the minimum mesh edge length was finally $0.12 \mu \mathrm{m}$, corresponding to $0.0004 \lambda$ at $1.0 \mathrm{THz}$. All of the performance characteristics and E-field distribution of each mode reported herein were attained with these settings. An $\exp (j w t)$ time dependence was assumed throughout.

\section{Slotted SPSM PCF Design Choices}

Two SPSM PCF designs are introduced as shown in Fig. 1. Both rely on a widely used, powerful approach to achieve HB PCFs, i.e., air slots are introduced into the core area [17]. They allow one to attain more exquisite control over the Efield distributions of the different modes. A rectangular gain area is set centrally between two adjacent rectangular air slots. The size of the gain-filled center slot is $L_{g} \times W_{g}$; the size of the air slots is $L_{a} \times W_{a}$. The distance between adjacent edges of the two air slots is $D$. Among these dimensions, the length of the air slots $L_{a}$ and width of the gain slot $W_{g}$ are the ones that has a significant coarse effect on the SPSM performance. All of the other dimensions provide a means to fine tune that performance.

High resistivity silicon (HRS) was selected to be the background material due to its outstanding properties in the $\mathrm{THz}$ regime, e.g., a low bulk material absorption loss: $<0.05$ $\mathrm{cm}^{-1}$ from 0.1-2.5 THz, and a flat refractive index: 3.417 from $0.5-4 \mathrm{THz}$ [21]. The ENZ material was set to have a 
constant refractive index of $n_{e n z}=0.2-0.1 j$ across the frequency range of interest. This ENZ property can be obtained with naturally existing materials or metamaterials [22-24]. The refractive index of the circular gain-impregnated region was set to be $n_{g}=3.417-\xi j$ where $\xi$ is the gain factor. It has the same relative permittivity as the background HRS material since the region was assumed to be only lightly doped with the gain material. Consequently, the gain regions are found to have only a minor effect on the real part of the refractive index due to the gain constant being small (i.e., only light doping is assumed). Gain materials such as rare earth materials (e.g., $\mathrm{Er}^{3+}$ ) [25] are well-established choices in optical fibers; nanoengineered Si:Er [26] and graphene [27] have been identified in the $\mathrm{THz}$ regime for loss compensation.

The performance of the SPSM PCF will be defined by two figures of merit, i.e., the SPSM bandwidth and the MLD value. The SPSM band is defined as the frequency range in which the TL of the XP mode is non-positive and the TLs of the YP and HO modes are positive. The MLD is defined as the minimum value of the LD (YP-XP) and LD (HO-XP).

\section{A. Type I PCF}

Fig. 1 gives the cross sectional views of the SPSM PCF candidate structures. As shown in Fig. 1(a), the general PCF structure is a triangular lattice distribution in the cladding, which is covered by a PML layer. As shown in Figs. 1(b) and 1(c), the ENZ material is deposited into four selected air holes in the first ring of the cladding, resulting in four ENZ rings. The selected ENZ-loaded air holes have diameter $d_{2}$ while all the other air holes have the diameter $d_{1}$. The distance between any two air holes is lattice constant $\Lambda$. This asymmetric cladding configuration can lead to different Efield distributions of the XP and YP modes, which results in different losses of the two fundamental modes. Two candidate SPSM structures are considered, labeled as Type I and Type II PCFs. They have different core regions. Zoom-in pictures of their core regions for both are given in Figs. 1(b) and 1(c). The details of the slots in both cores are shown in the shared dashed rectangular subplot.

Fig. 2(a) shows the E-field distributions of the XP, YP and higher order (HO) modes of the Type I slotted SPSM PCF in two cases. The lattice constant is set to be $\Lambda=70 \mu \mathrm{m}$. All the dimensions are then described in terms of it. The first has a short slot with $L_{a}=0.5 \Lambda$; the second has a long slot with $L_{a}$ $=0.8 \Lambda$. The remaining dimensions are fixed at $d_{1}=0.95 \Lambda, d_{2}$ $=0.83 \Lambda, L_{g}=0.5 \Lambda, W_{a}=0.14 \Lambda, W_{g}=0.08 \Lambda, D=0.456 \Lambda$, $\xi=-0.03$, and $t=0.18 \Lambda$. The PCF is now scalable to other frequencies by simply changing the value of $\Lambda$ according to the scaling property found in [20].

From Fig. 2(a), one observes that the E-field of the XP mode is highly localized in the core area while the E-field of the YP mode is concentrated in the air slots for both the long and short slot cases. Because the configuration is not symmetric along both the $\mathrm{X}$ and $\mathrm{Y}$ directions, the fundamental $\mathrm{X}$ and $\mathrm{Y}$ polarized modes have noticeably different $\mathrm{E}$-field distributions within the first ring region. The E-field of the XP mode is oriented primarily along the $\mathrm{x}$-axis and, hence,

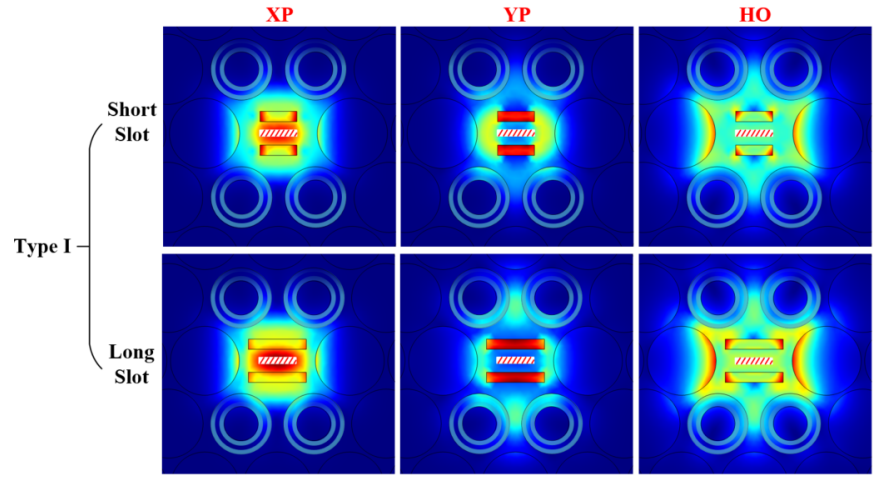

(a)

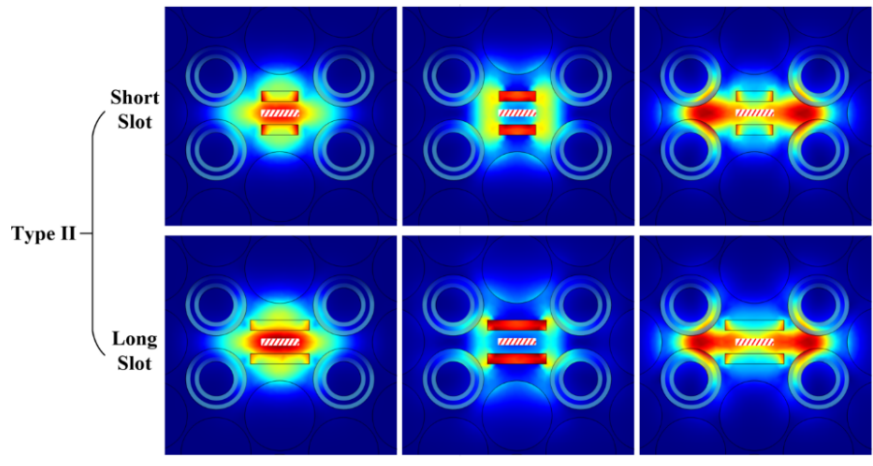

(b)

Fig. 2. Electric field distribution of the (a) Type I and (b) Type II slotted SPSM PCFs in two cases with short and long air slot lengths at $1.6 \mathrm{THz}$.

parallel to the slots while that of the YP mode is along the $\mathrm{y}$-axis and perpendicular to them. Thus, the electromagnetic interface condition for the E-field component of the YP mode normal to the slots forces large field values in them because of the large permittivity difference between the background dielectric and air $[18,20]$. Moreover, the E-field distribution of the XP mode, in contrast to that of the YP mode, is nearly uniform and concentrated in the region of the core between and along the slots. These E-field behaviors suggest that by introducing a rectangular gain area in the core center oriented the same as the air slots, a much larger amplification of the XP mode than of the YP mode will occur. This effect will naturally lead to larger LD values between the XP and YP modes. Furthermore, the E-field distribution of the $\mathrm{HO}$ mode shown in the figure is that of the lowest loss one among all of the HO modes. This HO mode is more concentrated near the lossy ENZ rings and less so in the core region. Consequently, it will experience smaller amplification from the gain area and a larger attenuation rate from the ENZ rings in comparison to the XP mode. These features will lead to desired large LD values between the XP and HO modes. With an overall optimization, this Type I slotted SPSM PCF achieves a SPSM bandwidth of $0.53 \mathrm{THz}$ with the MLD $>7.4 \mathrm{~dB}$, when $L_{a}=L_{g}=0.5 \Lambda$.

Furthermore, by comparing the E-field distributions of the long and short slot cases in Fig. 2(a), it is noticed that the longer slots effectively push the E-fields of the YP and HO modes away from the center of the core and into the airfilled slots. In contrast, the E-field distribution of the XP mode 
remains quite similar for either slot length. Consequently, it was decided that one could take advantage of very lossy ENZ rings in the circular air holes adjacent to the core along the long direction of the slots to introduce larger LDs between the wanted XP mode and all of the unwanted modes.

\section{B. Type II PCF}

The LD considerations of the Type I modes led to the Type II slotted SPSM PCF shown in Fig. 1(c), which rotated the cladding of the Type I configuration by $90^{\circ}$ and the core remains the same. However, the dimensions of the Type II PCF had to be re-optimized to maximize its performance because all of its modes have E-field distributions that are different from those of the Type I.

As shown in Fig. 2(b), the E-field distributions of the XP and YP modes for Type 2 PCF are quite similar to their Type 1 PCF counterparts. The different configurations barely affect their E-field distributions in the cladding region. On the other hand, the E-field of the YP mode within the first air-hole region is pushed closer to the ENZ rings when the longer air slots are introduced. Because the larger E-fields of the YP modes are distributed mainly in the region between the core and first airhole ring region, their apportionment changes when that first ring region changes. Since the E-fields of the $\mathrm{HO}$ modes are found to be "attracted" by the ENZ rings whether the air slots are short or long, this Type II design was expected to produce more losses to all of the unwanted modes. Comparing the Efield distributions in Figs. 2(a) and 2(b), it was then expected that the Type II PCF will exhibit both a larger LD (YP-XP) and LD (HO-XP) than those of the Type I PCF. Therefore, we selected the Type II slotted SPSM PCF to optimize as the desired configuration.

\section{TyPE II PCF DESIGN STUdiES}

Because of its expected improved performance, it was decided to focus efforts on the Type II PCF. While the achieved bandwidth of the Type II PCF was found to be much wider than the value attained with the Type I PCF, it was discovered that different $\mathrm{HO}$ modes appear over this wider frequency band and that they must be taken into consideration when optimizing the PCF, particularly to achieve the largest MLD. On the other hand, it was determined that an accurate MLD value is obtained if only the three $\mathrm{HO}$ modes with the lowest TL values are identified at a specific frequency. The difficulty is the fact that those three $\mathrm{HO}$ modes can be different at the lower, middle, and upper portions of the wide frequency band.

\section{A. Higher order mode behaviors}

Figs. 3(a) to 3(c) illustrate, as an example, the E-field distributions of the three major (smallest TL value) HO modes of the Type II slotted PCF with the long slot. Fig. 3(d) plots TLs of the XP, YP, and all the three HO modes as functions of the frequency. One observes immediately that there are "crossover" points at which each of the three identified HO modes have lower or higher TL values. Notice that the TL curve associated with the $\mathrm{HO}_{2}$ mode does not provide the MLD

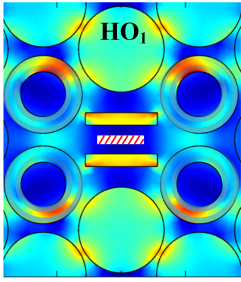

(a)

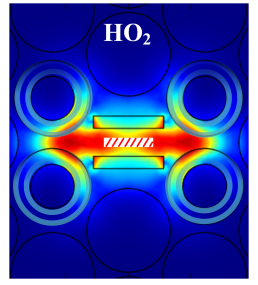

(b)

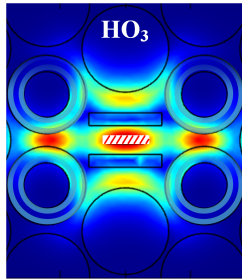

(c)

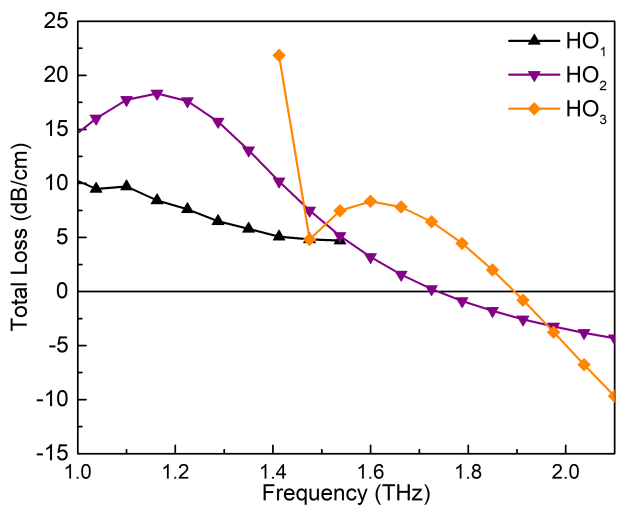

(d)

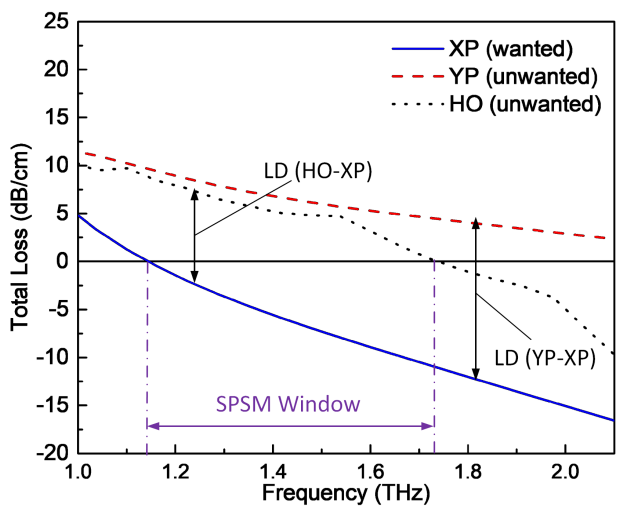

(e)

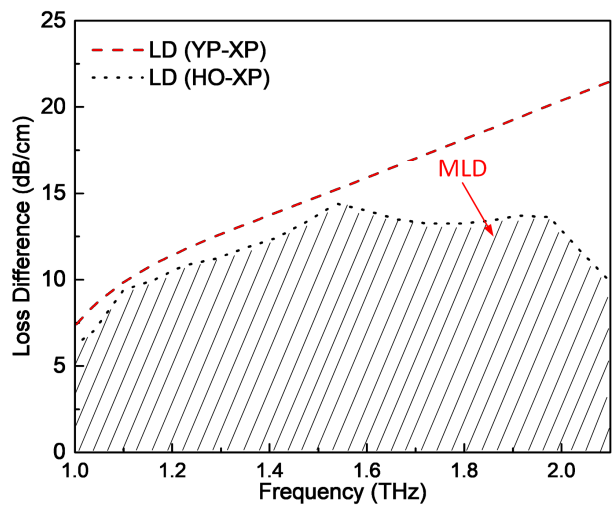

(f)

Fig. 3. Electric field distribution of $\mathrm{HO}$ modes of the Type II slotted PCF with the long slot. (a) $\mathrm{HO}_{1}$ at $1.2 \mathrm{THz}$. (b) $\mathrm{HO}_{2}$ at $1.6 \mathrm{THz}$. (c) $\mathrm{HO}_{3}$ at $2.0 \mathrm{THz}$. (d) The TL values of these three $\mathrm{HO}$ modes as functions of the source frequency. (e) The TL values of the XP and YP modes along with the composite (merged) lowest TL values of these three HO modes across the frequency band of interest. (f) The LD values between the YP and XP modes and between the $\mathrm{HO}$ and XP modes.

value at every frequency in the bandwidth. In contrast, the 
$\mathrm{HO}_{2}$ and $\mathrm{HO}_{3}$ modes have much higher losses in the lower frequency portion and the $\mathrm{HO}_{1}$ mode has the lowest LD values there. On the other hand, the $\mathrm{LD}$ values of the $\mathrm{HO}_{2}$ mode are the lowest in the middle portion of the band and those of the $\mathrm{HO}_{3}$ mode are the lowest in the upper portion. Returning to the field distributions in Figs. 3(a) to 3(c), one finds that one of the three modes shows the lowest loss in each portion of the bandwidth because it has some overlap with the gain region, while the other HO modes do not since they are not well confined in the center region. The three curves in Fig. 3(d) representing the TLs of the three $\mathrm{HO}$ modes are then merged into the single curve in Fig. 3(e). This composite curve thus represents the lowest TL value of all of the $\mathrm{HO}$ modes at each frequency in the operational band. These values will be denoted as the TL-HO values in the associated descriptions. As expected from the E-field distributions shown in Fig. 2(b), it is clearly observed that the wanted XP mode has the lowest TL compared to the unwanted YP and HO modes. Note that a TL value can be negative due to the presence of the gain material, i.e., the corresponding mode is amplified as it propagates. As defined previously, the SPSM window in this case is $0.59 \mathrm{THz}$, from 1.13 to $1.72 \mathrm{THz}$. Since the LD (HO-XP) has smaller values than that of LD (YP-XP) across the entire band, the MLD is determined by LD (HO-XP). In this case, the MLD is above $9.5 \mathrm{~dB} / \mathrm{cm}$ across the SPSM bandwidth.

To ensure that the MLD values were properly attained during the parametric studies performed to determine the optimal configuration, the LD values of the lowest $20 \mathrm{HO}$ modes were determined at each frequency in the band of interest. To simplify the presentation of those results, their composite (merged) result that signifies the MLD value at each frequency will be emphasized in the following discussions.

\section{B. Parametric Studies}

The Type II slotted SPSM PCF design parameters were investigated to optimize its performance characteristics. Since there are many, only the parametric studies of those having a key impact on the SPSM bandwidth and the MLD value are presented below. These include the gain factor $\xi$, the length of the air slots $L_{a}$, and the width of the gain area $W_{g}$. These studies will illustrate the effects of each parameter on the performance characteristics of the entire structure and the trade-off decisions made to determine their values for the overall final optimized design.

1) Gain factor $\xi$ : The gain factor $\xi$ is one of the most important design parameters. It determines the amount of amplification the gain area can provide to the modes overlapping with it. In practice, the size of $\xi$ is controlled by the doping ratio of the gain material [25]. Unfortunately, a large doping ratio increases the difficulty one would encounter in fabricating the PCF. Consequently, the gain factor was selected to have low values in these parameter studies.

The effects on the SPSM bandwidth and MLD value when $\xi$ was varied from -0.030 to -0.040 with a step size of 0.005 were studied. During this parameter sweep, the other parameters were fixed as: $d_{1}=0.95 \Lambda, d_{2}=0.86 \Lambda, L_{a}=$ $0.7 \Lambda, L_{g}=0.4 \Lambda, W_{a}=0.14 \Lambda, W_{g}=0.11 \Lambda, D=0.456 \Lambda$,

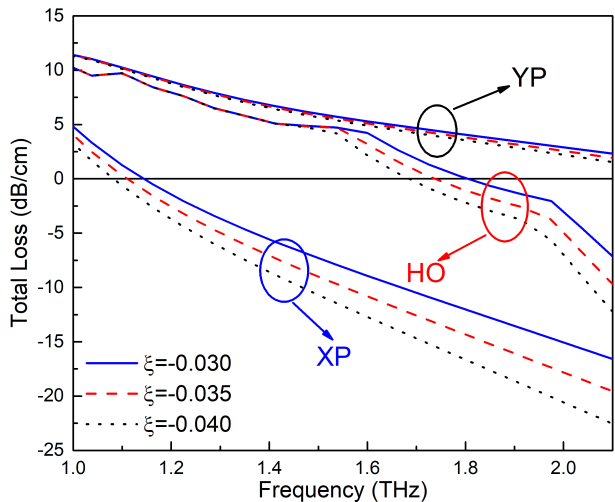

(a)

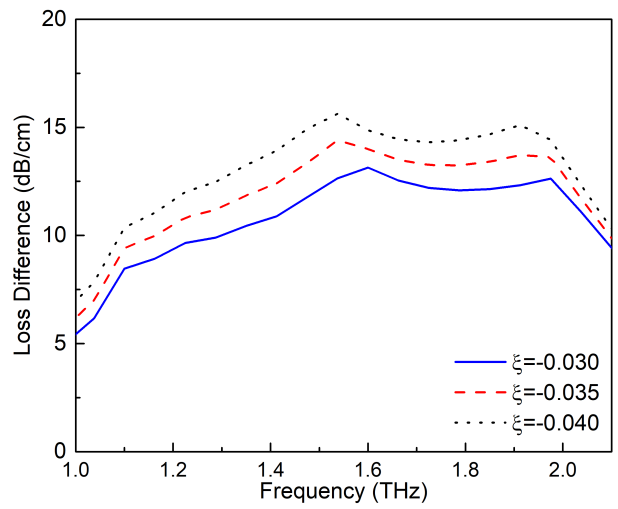

(b)

Fig. 4. (a) Simulated TL values of the XP, YP, and HO modes of the Type II slotted SPSM PCF as functions of the source frequency for three different gain factor, $\xi$, values. (b) The corresponding composite curves indicating the smallest LD values between the wanted and unwanted modes over the frequency band of interest.

$t=0.15 \Lambda$, and $\Lambda=70 \mu \mathrm{m}$. The resulting simulated TL values of the different modes in this fixed geometry for the different $\xi$ values are shown in Fig. 4(a) as functions of the source frequency. The ability of the gain area to amplify the local E-field distribution clearly strengthens as $|\xi|$ increases. As a consequence, the TL values of the XP mode decrease because its E-field is well confined in the gain area. The YP mode, however, is not affected because its E-field is mainly confined in the air slots as illustrated in Fig. 2(b). Similarly, the TL values of the $\mathrm{HO}$ modes remain fairly stable at the lower frequencies as $|\xi|$ increases. As shown in Fig. 3(a), the $\mathrm{HO}_{1}$ mode has the lowest loss at those frequencies and it only has a small portion of its E-field distributed over the gain area. Its TL values dominate the TL-HO ones at these lower frequencies. On the other hand, as already noted and illustrated in Figs. 3(b) and $3(\mathrm{c})$, the TLs of the $\mathrm{HO}_{2}$ and $\mathrm{HO}_{3}$ modes dominate the composite TL-HO values at the middle and higher frequencies, respectively, because they have more overlap with the gain area. Accounting for the TL variations of all of the modes, the smallest LD values between the wanted and unwanted modes were obtained. These composite LD values are plotted in Fig. 4(b) for the three different gain factors.

Consider now the crossover points, i.e., the frequency point at which the TL value of the wanted mode becomes zero and 
TABLE I

CROSSOVER POINTS, SPSM BANDWIDTH, AND MLD VALUES FOR DIFFERENT GAIN FACTORS $\xi$.

\begin{tabular}{ccccc}
\hline$\xi$ & $\begin{array}{c}\text { Crossover } \\
\text { wanted mode } \\
(\mathrm{THz})\end{array}$ & $\begin{array}{c}\text { Crossover } \\
\text { unwanted mode } \\
(\mathrm{THz})\end{array}$ & $\begin{array}{c}\text { SPSM } \\
\text { Bandwidth } \\
(\mathrm{THz})\end{array}$ & $\begin{array}{c}\text { MLD } \\
(\mathrm{dB} / \mathrm{cm})\end{array}$ \\
\hline-0.030 & 1.14 & 1.80 & 0.66 & $>8.8$ \\
-0.035 & 1.10 & 1.74 & 0.64 & $>9.4$ \\
-0.040 & 1.08 & 1.68 & 0.60 & $>9.5$ \\
\hline
\end{tabular}

the higher frequency point at which the lowest TL value of all of the unwanted modes becomes zero. These two crossover points define the SPSM bandwidth. As $|\xi|$ increases, they both shift towards a lower frequency. Table I summarizes the variations of these crossover points, the resultant SPSM bandwidths, and the MLD value over the SPSM band with different $\xi$. In this work, $\xi$ was selected to be -0.035 . It has a reasonable trade-off between the SPSM bandwidth and the MLD value. Note that the unities for crossover, bandwidth, and MLD in all tables of this paper are $\mathrm{THz}, \mathrm{THz}$ and $\mathrm{dB} / \mathrm{cm}$, respectively.

2) Length of air slots $L_{a}$ : Another important design parameter is the length of the air slots $L_{a}$. As already demonstrated in Fig. 2, the length of the air slots has a significant effect on the E-field distributions of the unwanted modes. A parameter study of $L_{a}$ was conducted by sweeping its value from $0.6 \Lambda$ to $0.8 \Lambda$ and determining its impact on the SPSM bandwidth and the MLD value. All of the other design parameters were fixed at: $d_{1}=0.95 \Lambda, d_{2}=0.86 \Lambda, L_{g}=0.4 \Lambda, W_{a}=0.14 \Lambda$, $W_{g}=0.11 \Lambda, D=0.456 \Lambda, \xi=-0.035, t=0.15 \Lambda$, and $\Lambda=$ $70 \mu \mathrm{m}$.

Fig. 5(a) presents the simulated TLs of the XP, YP, and HO modes for the different values of $L_{a}$ as functions of the source frequency. The variations of the LDs between the wanted and unwanted modes with frequency are presented in Fig. 5(b). As shown in Fig. 5(a), the TLs of the XP mode remain quite stable across the frequency band of interest. On the other hand, the TLs of the YP mode increase as $L_{a}$ increases. This behavior was expected from Fig. 2(b) which showed that increasing the length of the air slots does not change the E-field distribution of the XP mode, while the longer air slots pushed the E-field of the YP mode into the ENZ rings. This effect clearly leads to the observed higher TLs of the YP mode. Furthermore, $L_{a}$ only has a minimal effect on the TL values of the HO modes since the three significant ones have only very small portions of their E-fields distributed in the air slots. The resultant composite smallest $\mathrm{LD}$ values between the wanted mode and the unwanted modes are shown in Fig. 5(b).

The crossover points, the SPSM bandwidth, and the MLD values for different values of $L_{a}$ are listed in Table 2. Since the $L_{a}=0.7 \Lambda$ case produced the widest SPSM bandwidth and the highest MLD value, it was selected to be the optimal value.

3) Width of gain area $W_{g}$ : As emphasized, the gain area has to be optimized to maximize the amplification to the wanted mode and to minimize its amplification of the unwanted modes. One key design parameter that impacts of the

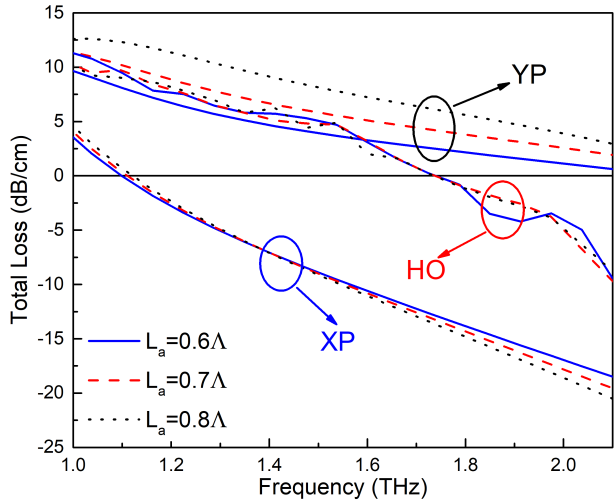

(a)

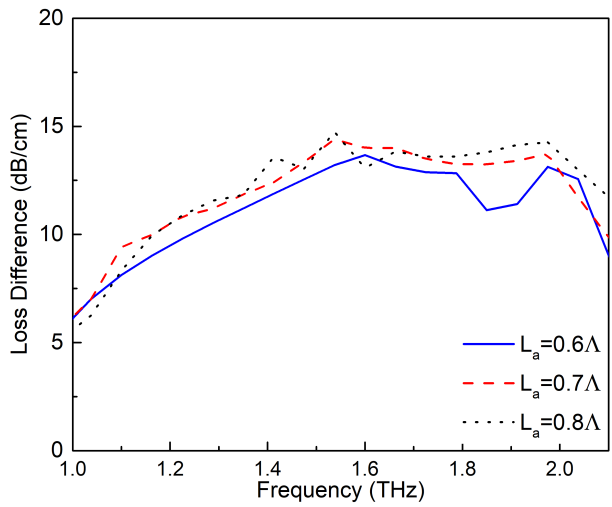

(b)

Fig. 5. (a) Simulated TL values of the XP, YP, and HO modes of the Type II slotted SPSM PCF as functions of the source frequency for three different air slots lengths, $L_{a}$. (b) The corresponding composite curves indicating the smallest LD values between the wanted and unwanted modes over the frequency band of interest.

TABLE II

CROSSOVER POINTS, SPSM BANDWIDTH, AND MLD VALUES FOR DIFFERENT AIR SLOT LENGTH $L_{a}$.

\begin{tabular}{ccccc}
\hline$L_{a}$ & $\begin{array}{c}\text { Crossover } \\
\text { wanted mode } \\
(\mathrm{THz})\end{array}$ & $\begin{array}{c}\text { Crossover } \\
\text { unwanted mode } \\
(\mathrm{THz})\end{array}$ & $\begin{array}{c}\text { SPSM } \\
\text { Bandwidth } \\
(\mathrm{THz})\end{array}$ & $\begin{array}{c}\text { MLD } \\
(\mathrm{dB} / \mathrm{cm})\end{array}$ \\
\hline $0.6 \Lambda$ & 1.16 & 1.74 & 0.66 & $>9.0$ \\
$0.7 \Lambda$ & 1.10 & 1.74 & 0.64 & $>9.4$ \\
$0.8 \Lambda$ & 1.12 & 1.74 & 0.60 & $>8.8$ \\
\hline
\end{tabular}

gain area is the width, $W_{g}$, of the gain impregnated slot. The parameter $W_{g}$ was varied from $0.07 \Lambda$ to $0.13 \Lambda$ to determine its effects on the bandwidth and MLD values. During the parameter sweep, the other parameters were fixed as: $d_{1}=$ $0.95 \Lambda, d_{2}=0.86 \Lambda, L_{a}=0.7 \Lambda, L_{g}=0.4 \Lambda, W_{a}=0.14 \Lambda, D$ $=0.456 \Lambda, \xi=-0.035, t=0.15 \Lambda$, and $\Lambda=70 \mu \mathrm{m}$.

Figs. 6(a) and 6(b) present, respectively, the simulated TL and $L D$ values of the wanted and unwanted modes as functions of the source frequency for different values of $W_{g}$. As shown in Fig. 6(a), the variation of $W_{g}$ leads to significant changes to the TL values of the XP mode. Re-inspecting the E-field distribution of the XP mode of the PCF shown in Fig. 2(b), one finds that with a wider gain area, a larger portion of E-field is located in it. Consequently, lower TL values are 


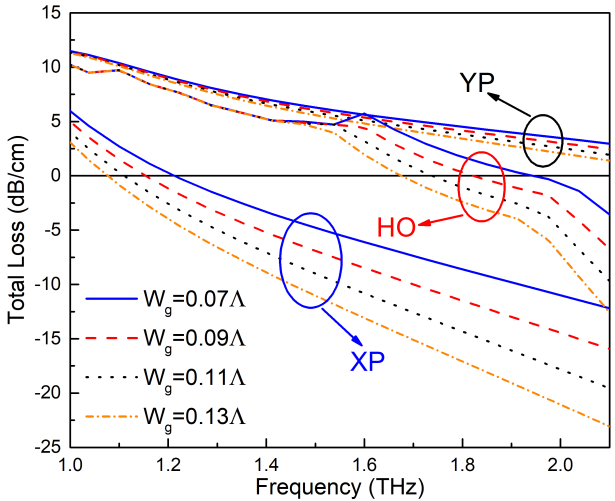

(a)

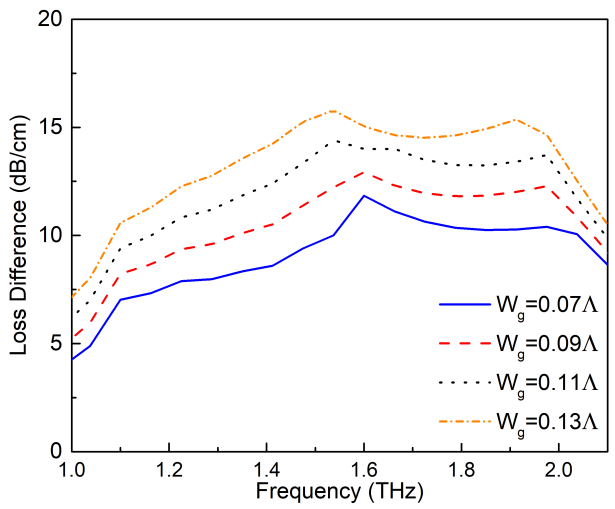

(b)

Fig. 6. (a) Simulated TL values of the XP, YP, and HO modes of the Type II slotted SPSM PCF as functions of the source frequency for four different gain area widths, $W_{g}$. (b) The corresponding composite curves indicating the smallest LD values between the wanted and unwanted modes over the frequency band of interest.

attained. The YP mode, however, experiences less effect with variations in $W_{g}$ because its E-field is mainly concentrated in the air slots. Similarly, the increase of $W_{g}$ has only minimal impact on the TL-HO values at the lower frequencies where the $\mathrm{HO}_{1}$ dominates the others $\mathrm{HO}$ modes. As shown in Fig. 3(a), the $\mathrm{HO}_{1}$ mode has only a very small portion of its $\mathrm{E}$ field distributed overlapping the gain area. However, the TL$\mathrm{HO}$ values are affected at the higher frequencies where, as shown in Figs. 3(b) and 3(c), the $\mathrm{HO}_{2}$ or $\mathrm{HO}_{3}$ modes have some overlap with the gain area and, hence, dominate the TL behavior there. The TL-HO values thus decrease with an increase of $W_{g}$ at those higher frequencies. Given the variations of the TLs of all of the modes with $W_{g}$, the composite smallest $\mathrm{LD}$ values between the wanted mode and the unwanted modes were obtained and are plotted in Fig. 6(b).

The crossover points, the SPSM bandwidth, and the MLD values for different values of the gain slot width, $W_{g}$, are summarized in Table 3. The width of the gain slot, $W_{g}=$ $0.11 \Lambda$, was selected as the optimal value because it produced the largest MLD while maintaining a reasonable SPSM bandwidth. Note that an even larger MLD could be achieved if one were willing to sacrifice some bandwidth.

4) Optimized results: In addition to the parametric sweeps explicitly presented in the previous subsections, we have also
TABLE III

CROSSOVER POINTS, SPSM BANDWIDTH, AND MLD VALUES FOR DIFFERENT GAIN AREA WIDTH $W_{g}$.

\begin{tabular}{ccccc}
\hline$W_{g}$ & $\begin{array}{c}\text { Crossover } \\
\text { wanted mode } \\
(\mathrm{THz})\end{array}$ & $\begin{array}{c}\text { Crossover } \\
\text { unwanted mode } \\
(\mathrm{THz})\end{array}$ & $\begin{array}{c}\text { SPSM } \\
\text { Bandwidth } \\
(\mathrm{THz})\end{array}$ & $\begin{array}{c}\text { MLD } \\
(\mathrm{dB} / \mathrm{cm})\end{array}$ \\
\hline $0.07 \Lambda$ & 1.21 & 1.94 & 0.73 & $>7.8$ \\
$0.09 \Lambda$ & 1.15 & 1.82 & 0.67 & $>8.6$ \\
$0.11 \Lambda$ & 1.10 & 1.74 & 0.64 & $>9.4$ \\
$0.13 \Lambda$ & 1.07 & 1.68 & 0.61 & $>9.4$ \\
\hline
\end{tabular}

TABLE IV

PERFORMANCE COMPARISON BETWEEN THE PREVIOUSLY REPORTED THZ SPSM PCFS AND THE ONES DEVELOPED IN THIS PAPER.

\begin{tabular}{ccc}
\hline Works & $\begin{array}{c}\text { SPSM Bandwidth } \\
(\mathrm{THz})\end{array}$ & $\begin{array}{c}\text { MLD } \\
(\mathrm{dB} / \mathrm{cm})\end{array}$ \\
\hline$[15]$ & 0.13 & $>0.036$ \\
{$[17]$} & 0.32 & $>0.06$ \\
{$[18]$} & 0.31 & $>8.0$ \\
Type I & 0.53 & $>7.4$ \\
Type II & $\mathbf{0 . 6 4}$ & $>\mathbf{9 . 4}$ \\
\hline
\end{tabular}

conducted a set of parameter studies for several other design parameters, e.g., the thickness of the ENZ rings $t$, the length of the gain slot $L_{g}$, the width of the air slots $W_{a}$, and the distance $D$ between the slots in the core region. It was determined that the ENZ ring thickness only has minor effects on the performance because the E-field distributions of the various modes are dominated by the air slots rather than the ENZ rings. The ENZ rings introduce the largest losses into the configuration. The associated attenuation rate of any mode is determined primarily by the imaginary part of the relative permittivity of the ENZ material in those rings, rather than their thickness. The other design parameters show trade-off effects similar to those found with $W_{g}$. One may have to give up some bandwidth (MLD) to enhance the MLD (bandwidth) value. To streamline the presentation, these parameter studies were not presented.

All of these parametric studies led to the final overall optimized Type II SPSM PCF design. Its parameters are: $d_{1}$ $=0.95 \Lambda, d_{2}=0.86 \Lambda, L_{a}=0.7 \Lambda, L_{g}=0.4 \Lambda, W_{a}=0.14 \Lambda$, $W_{g}=0.11 \Lambda, D=0.456 \Lambda, \xi=-0.035, t=0.15 \Lambda$, and $\Lambda=$ $70 \mu \mathrm{m}$. This optimized PCF design has an ultra-wide SPSM bandwidth of $0.64 \mathrm{THz}$ from 1.10 to $1.74 \mathrm{THz}$ and a very large MLD $>9.4 \mathrm{~dB} / \mathrm{cm}$ over its entire operational bandwidth. The superiority of the main performance characteristics of this final design are demonstrated by the comparisons with the state-ofart THz SPSM PCFs reported in the literature given in Table 4. In summary, remarkable performance improvements were achieved in comparison to previous designs. For the Type I PCF, the bandwidth has been enhanced by $70 \%$ with only a minor sacrifice of the MLD value compared to the design reported in [18]. For the Type II PCF, the bandwidth was increased by more than a factor of two and the MLD value was increase by $17.5 \%$. 


\section{Fabrication issues}

While we do not have access to the means to accomplish and test a prototype of our design, we are nevertheless quite confident that one can be realized with existing technologies, particularly for the dimensions associated with a $\mathrm{THz}$ structured fiber. A conventional approach for optical fibers is to construct an appropriate fiber preform which could be done by a stacking process [6], drilling [28], or die extrusion [8]. The preform would then be put it into the fiber-drawing tower and the PCF would be drawn under a specialized pressure environment into the required dimensions. A more recent approach is to use 3D printing techniques. Successful 3D printed preforms and the corresponding drawn microstructured optical fibers have been reported [29-31]. All of these methods can be applied to realize a prototype of our SPSM PCF design. In particular, the previously reported 3D printing works, e.g., [32], [33], have demonstrated microstructured THz fibers with feature dimensions immediately relevant to our SPSM PCF design.

Although the proposed PCF design itself should, therefore, not be too difficult to fabricate, the integration of the ENZ rings into four specific air holes must also be accomplished. Hybrid optical fiber techniques involving more complex structures [34] and material compositions [35], [36] are immediately relevant. For instance, one could insert naturally occurring bulk materials with ENZ properties such as KCL [22] into the desired mircostructured fiber holes with direct thermal drawing [37] or pressure-assisted melt filling [38]. Moreover, because the requisite thickness of the ENZ rings is very small, these thin layers could also be accomplished with the high-pressure chemical vapor deposition [39-41] of semiconductor plasmonic materials [22]. Alternatively, specifically engineered metamaterials could be integrated into the fiber [34], [35] instead of naturally occurring ENZ materials to attain the desired ENZ properties at the requisite frequencies. Furthermore, with the increasing attention paid to ENZ materials [42-44] and hybrid microstructured fibers [34], [35], we believe that more naturally and artificially realized ENZ materials, along with more advanced fabrication techniques, will be available to successfully realize our SPSM PCF design in the near future.

One would expect that imperfections introduced during the fabrication of any optimized design would have an impact on its performance characteristics. However, the parametric studies discussed above suggest that the performance of our optimized Type II SPSM PCF design is relatively stable even with significant changes to its key design parameters. Further parametric studies indicate that it is quite robust and should have a high tolerance to any fabrication issues.

It is also fully recognized that realistic ENZ materials are dispersive. Even though the overall percentage of material represented by the ENZ rings in the structure is small, they play a significant role in the operational mechanisms, notably the losses they facilitate near the core region. A study of the optimized design with dispersive ENZ rings was undertaken.

Recall that the optimized structure is based on the refractive index of the ENZ material being fixed at $n_{e n z}=0.2-$
TABLE V

CROSSOVER POINTS, SPSM BANDWIDTH, AND MLD VALUES FOR DIFFERENT REAL PART VALUES OF $n_{e n z}$ WHEN ITS IMAGINARY PART IS FIXED AT $0.1 j$.

\begin{tabular}{ccccc}
\hline $\operatorname{Re}\left(n_{\text {enz }}\right)$ & $\begin{array}{c}\text { Crossover } \\
\text { wanted mode } \\
(\mathrm{THz})\end{array}$ & $\begin{array}{c}\text { Crossover } \\
\text { unwanted mode } \\
(\mathrm{THz})\end{array}$ & $\begin{array}{c}\text { SPSM } \\
\text { Bandwidth } \\
(\mathrm{THz})\end{array}$ & MLD \\
$(\mathrm{dB} / \mathrm{cm})$
\end{tabular}

TABLE VI

CROSSOVER POINTS, SPSM BANDWIDTH, AND MLD VALUES FOR DIFFERENT IMAGINARY PART VALUES OF $n_{e n z}$ WHEN ITS REAL PART IS FIXED AT 0.2 .

\begin{tabular}{ccccc}
\hline $\operatorname{Im}\left(n_{\text {enz }}\right)$ & $\begin{array}{c}\text { Crossover } \\
\text { wanted mode } \\
(\mathrm{THz})\end{array}$ & $\begin{array}{c}\text { Crossover } \\
\text { unwanted mode } \\
(\mathrm{THz})\end{array}$ & $\begin{array}{c}\text { SPSM } \\
\text { Bandwidth } \\
(\mathrm{THz})\end{array}$ & MLD \\
\hline 0.050 & 0.97 & 1.50 & 0.53 & $>5.6$ \\
0.075 & 1.05 & 1.63 & 0.58 & $>8.0$ \\
0.100 & 1.10 & 1.74 & 0.64 & $>9.4$ \\
0.150 & 1.21 & 1.93 & 0.72 & $>9.3$ \\
0.200 & 1.29 & 2.08 & 0.79 & $>8.1$ \\
\hline
\end{tabular}

$0.1 j$. However, both the real and imaginary parts of $n_{e n z}$ can change with frequency over the wide bandwidth of operation, i.e., from 1.10 to $1.74 \mathrm{THz}$. Therefore, different real and imaginary parts of $n_{e n z}$ were employed to calculate the associated variations experienced by the SPSM bandwidth and MLD values. The results are summarized in Tables 5 and 6, respectively. One observes that as the real or imaginary parts of $n_{e n z}$ increases, the achieved SPSM bandwidth becomes wider, but shifts to higher frequency. The MLD value generally increases as well. These parametric studies clearly indicate that the performance variations resulting from dispersion in the ENZ material is reasonable and within an acceptable range.

\section{CONCLUSION}

A THz SPSM PCF design was developed in this paper that yields an ultra-wide bandwidth over which SPSM propagation occurs with large MLD values between the wanted (XP) and unwanted (YP and HO) modes. The significant enhancement of the SPSM bandwidth and MLD values was facilitated by a custom-designed core region. Three rectangular slots were introduced in the central region of the core and the middle slot was loaded with gain material. Furthermore, ENZ material rings were introduced in four selected air holes surrounding the core region. The configuration was optimized with extensive parametric studies of the design parameters and the subsequent behaviors of the wanted and unwanted modes. The operating mechanisms and optimization path were illustrated with a variety of simulation results. It was demonstrated that the optimized design achieves an ultra-wide SPSM bandwidth of $0.64 \mathrm{THz}$, from 1.10 to $1.74 \mathrm{THz}$, with a high MLD value of $9.4 \mathrm{~dB} / \mathrm{cm}$. With these attractive performance characteristics, the reported PCF is a competitive candidate for a variety of $\mathrm{THz}$ wave guiding applications requiring polarization purity. 
In particular, it can achieve a single polarization state within a short propagation distance, e.g., the wanted mode becomes $30 \mathrm{~dB}$ higher than the unwanted modes after propagating in only a $3.2 \mathrm{~cm}$ long segment of the optimized SPSM PCF.

\section{REFERENCES}

[1] I. F. Akyildiz, J. M. Jornet, C. Han, ’Terahertz band: Next frontier for wireless communications," Phys. Com., vol.12, pp.16-32, Sep. 2014.

[2] A. Redo-Sanchez and X.-C. Zhang, "Terahertz science and technology trends," IEEE J. Sel. Top. Quantum Electron., vol. 14, no. 2, pp. 260269, Apr. 2008.

[3] N. Riesen, J. D. Love, and J. W. Arkwright, "Few-mode ellipticalcore fiber data transmission," IEEE Photon. Technol. Lett., vol. 24, no. 5, pp. 344-346, Mar. 2012.

[4] H. Yan, S. Li, Z. Xie, X. Zhang, H. Zhang, and B. Zhou, "Design of PANDA ring-core fiber with 10 polarization-maintaining modes," Photon. Res., vol. 5, no. 1, pp. 1-5, Feb. 2017

[5] K. A. Murphy, M. S. Miller, A. M. Vengsarkar, and R. O. Claus, "Elliptica-core two-mode optical-fiber sensor implementation methods," J. Lightw. Technol., vol. 8, no. 11, pp. 1688-1696, Nov. 1990.

[6] P. St. J. Russell, "Photonic-crystal fibers," J. Lightw. Technol., vol. 24 no. 12, pp. 4729-4749, Dec. 2006 .

[7] T. Yang, E. Wang, H. Jiang, Z. Hu, and K. Xie, "High birefringence photonic crystal fiber with high nonlinearity and low confinement loss," Opt. Express, vol. 23, no. 7, pp. 8329-8337, Mar 2015.

[8] S. Atakaramians, S. Afshar V, H. Ebendorff-Heidepriem, M. Nagel, B. M. Fischer, D. Abbott, and T. M. Monro, "THz porous fibers: design, fabrication and experimental characterization," Opt. Express, vol. 17, no. 16, pp. 14053-15062, Aug. 2009.

[9] G. K. M. Hasanuzzaman, S. Rana, and M. S. Habib, "A novel low loss, highly birefringent photonic crystal fiber in THz regime," IEEE Photon. Technol. Lett., vol. 28, no. 8, pp. 899-902, Apr. 2016.

[10] T.-Y, Yang, C. Ding, R. W. Ziolkowski, Y. J. Guo, "Circular hole ENZ photonic crystal fibers exhibit high birefringence," Opt. Express, vol. 26, no .13, pp. 17264-17278, Aug. 2018.

[11] R. Sohel, A. S. Rakin, H. Subbaraman, R. Leonhardt, and D. Abbott, "Low loss and low dispersion fiber for transmission applications in the terahertz regime," IEEE Photon. Techn. Letts., vol. 29, no. 10, pp. 830833, 2017.

[12] R. Sohel, A. S. Rakin, M. R. Hasan, M. S. Reza, R. Leonhardt, D. Abbott, and H. Subbaraman, "Low loss and flat dispersion Kagome photonic crystal fiber in the terahertz regime," Op. Commun., vol. 410 , pp. 452-456, 2018.

[13] R. Sohel, M. S. Islam, M. Faisal, K. C. Roy, R. Islam, and S. F. Kaijage, "Single-mode porous fiber for low-loss polarization maintaining terahertz transmission," Opt. Engineering, vol. 55, no. 7, pp. 076114, 2016.

[14] M.-Y. Chen, B. Sun, and Y.-K. Zhang, "Broadband single-polarization operation in square-lattice photonic crystal fibers," J. Lightw. Technol., vol. 28, no. 10, pp. 1443-1446, May 2010.

[15] Y. Huo, F. Fan, H. Zhang, X. Wang, and S. Chang, "Terahertz singlepolarization single-mode hollow-core fiber based on index-matching coupling," IEEE Photon. Techno. Lett., vol. 24, no. 8, pp. 637-639, Apr. 2012

[16] K. Satioh and M. Koshiba, "Single-polarization single-mode photonic crystal fibers," IEEE Photon. Technol. Lett., vol. 15, no. 10, pp. 13841386, Oct. 2003

[17] H. Chen, H. Wang, H. Hou, and D. Chen, "A terahertz singlepolarization single-mode photonic crystal fiber with a rectangular array of micro-holes in the core region," Opt. Comm., vol. 285, no. 18, pp. 3726-3729, Aug. 2012

[18] T.-Y. Yang, C. Ding, R. W. Ziolkowski, Y. J. Guo, ”A terahertz (THz) single-polarization-single-mode (SPSM) photonic crystal fiber (PCF)," Materials, vol. 12, no. 15, pp. 2442, Jul. 2019.

[19] COMSOL Multiphysics version 5.3, 2018. COMSOL AB, Stockholm. Available at: http://cn.comsol.com/rf-module.

[20] T.-Y, Yang, C. Ding, R. W. Ziolkowski, Y. J. Guo, ”A scalable THz ultrahigh birefringence and ultra-low loss partially-slotted photonic crystal fiber," IEEE J. Lightw. Technol., vol. 36, no .16, pp. 3408-3417, Aug. 2018.

[21] J. Dai, J. Q. Zhang, W. L. Zhang, and D. Grischkowsky, ’Terahertz time-domain spectroscopy characterization of the far-infrared absorption and index of refraction of high-resistivity, float-zone silicon," Opt. Soc. Am. B, vol. 21, no.7, pp. 1379-1386, July 2004.
[22] A. Reyes-Coronado, M. F. Acosta, R. I. Merino, V. M. Orera, G Kenanakis, N. Katsarakis, M. Kafesaki, C. Mavidis, A. J. Garcia, E. N. Economou, and C. M. Soukoulis, "Self-organization approach for THz polaritonic metamaterials," Opti. Express, vol. 20, no. 12, pp. 1466314682, Jun. 2012

[23] G. V. Naik, V. M. Shalaev, and A. Boltasseva, "Alternative plasmonic materials: beyond gold and silver," Adv. Mater, vol. 25, pp. 3264-3294, Jun. 2013.

[24] Engheta N, Ziolkowski R W. Metamaterials: Physics and Engineering Explorations. New Jersy, John Wiley \& Sons, 2006.

[25] Z. M. Sathi, J. Zhang, Y. Luo, J. Canning, and G. D. Peng, 'Improving broadband emission within $\mathrm{Bi} / \mathrm{Er}$ doped silicate fibres with $\mathrm{Yb}$ codoping," Opt. Express, vol. 5, no. 10, pp. 2096-2105, Oct. 2015

[26] N. Q. Vinh, N. N. Ha, and T. Gregorkiewicz, "Photonic properties of Er-doped crystalline silicon," Proc. of the IEEE, vol. 97, no. 7, pp. 12691283, Jul. 2009.

[27] F. Rana, "Graphene terahertz plasmon oscillators," IEEE Trans. Nanotechnol., vol. 7, no. 1, pp. 91-99, Jan. 2008.

[28] K. Nielsen, H. K. Rasmussen, A. J. L. Adam, P. C. M. Planken, O. Bang, and P. U. Jepsen, "Bendable, low-loss Topas fibers for the terahertz frequency range," Opt. Express, vol. 17, no. 10, pp. 8592-8601, 2009.

[29] K. Cook, J. Canning, S. Leon-Saval, Z. Reid, Md A. Hossain, J.-E. C., Y. Luo, and G.-D. Peng, "Air-structured optical fiber drawn from a 3D-printed preform," Opt. Lett., vol. 40, no. 17, pp. 3966-3969, 2015.

[30] J. Canning, Md. A. Hossain, C. Han, L. Chartier, K. Cook, and T. Athanaze, "Drawing optical fibers from three-dimensional printers," Opt. Lett., vol. 41, no. 23, pp. 5551-5554, 2016.

[31] Y. Chu, X. Fu, Y. Luo, J. Canning, Y. Tian, K. Cook, J. Zhang, and G.-D. Peng, "Silica optical fiber drawn from 3D printed preforms," Opt. Lett., vol. 44, no. 21, pp. 5358-5361, 2019.

[32] Z.-R Wu, W.-R. Ng, M. E. Gehm, and H. Xin, "Terahertz electromagnetic crystal waveguide fabricated by polymer jetting rapid prototyping," Opt. Express, vol. 19, no. 5, pp. 3962-3972, Feb. 2011.

[33] H. Xin, and M. Liang, "3-D-printed microwave and THz devices using polymer jetting techniques, "Proc. IEEE, vol. 105, no. 4, pp. 737-755, 2017.

[34] M. A. Schmidt, A. Argyros, and F. Sorin, "Hybrid optical fiber - an innovative platform for in-fiber photonic devices," Adv. Optical Mater. vol. 4 , no. 1 , pp. 13-36, 2016.

[35] C. Markos, J. C. Travers, A. Abdolvand, B. J. Eggleton, O. Bang, "Hybrid photonic-crystal fiber," Rev. Mod. Phys., vol. 89, no. 4, pp. 045003, 2017.

[36] V. Pacheco-Pena, N. Engheta, S. Kuznetsov, A. Gentselev, and M. Beruete, "Experimental realization of an epsilon-near-zero graded-index metalens at terahertz frequencies," Phys. Rev. Appl., vol. 8, no. 3, pp. 034036, 2017.

[37] J. Hou, D. Bird, A. George, S. Maier, B. T. Kuhlmey, and J. C. Knight, "Metallic mode confinement in mircrostructured fibres," Opt. Express, vol. 16, no. 9, pp. 5983-5990, Apr. 2008.

[38] H. W. Lee, M. A. Schmidt, R. F. Russell, n. Y. Joly, H. K. Tyagi, P. Uebel, and P. St. J. Russell, "Pressure-assisted melt-filling and optical characterization of Au nano-wires in microstructured fibers," Opt. Express, vol. 19, no. 13, pp. 12180-12189, Jun. 2011.

[39] A. Amezcua-Correa, J. Yang, C. E. Finlayson, A. C. Peacock, J. R. Hayes, P. J. A. Sazio, J. J. Baumberg, and S. M.Howdle, "Surfaceenhanced raman scattering using microstructured optical fiber substrates," Adv. Funct. Mater., vol. 17, no. 13, pp. 2024-2030, 2007.

[40] N. F. Baril, R. He, T. D. Day, J. R. Sparks, B. Keshavarzi, M. Krishnamurthi, A. Borhan, V. Gopalan, A. C. Peacock, N. Healy, P. J. A. Szio, and J. V. Badding, "Confined high-pressure chemical deposition of hydrogenated amorphous silicon," J. Am. Chem. Soc., vol. 134, no. 1, pp. 19-22, 2012.

[41] R. He, P. J. A. Sazio, A. C. Peacock, N. Healy, J. R. Sparks, M. Krishnamurthi, V. Gopalan, and J. V. Badding, "Integration of gigahertzbandwidth semiconductor devices inside microstructured optical fibres," Nature Photonics, vol. 6, pp. 174-179, 2012.

[42] G. V. Naik and A. Boltasseva, "A comparative study of semiconductorbased plasmonic metamaterials," Metamaterials, vol. 5, no. 1, pp. 1-7, 2011.

[43] I. Liberal and N. Engheta, "Zero-index platforms: Where light defies geometry," Opt. Photonics News, vol. 27, no. 7, pp. 26-33, 2016.

[44] I. Liberal and N. Engheta, "Near-zero refractive index photonics," Nat. Photonics, vol. 11, pp. 149-158, 2017. 
Tianyu Yang was born in Anhui Province, China, in 1990. He received a Bachelor degree and Master degree in Measurement and Control Technology from Hefei University of Technology, Hefei, China, in 2012 and 2016 respectively. He is currently working toward the Ph.D. degree in Engineering at University of Technology Sydney (UTS), Sydney, Australia. He has authored/co-authored 10 papers in peer-reviewed international journals. His current research interests include $\mathrm{THz}$ and optical crystal fiber design and its sensing application.

Can Ding (M'2016) received the bachelor's degree in microelectronics from Xidian University, Xi'an, China, in 2009, and the Ph.D. degree from Macquarie University, Sydney, Australia, in 2015. From 2012 to 2015, he was under the cotutelle agreement between Macquarie University and Xidian University. During this samesss period, he was also with Commonwealth Scientific and Industrial Research Organisation DPaS Flagship, Marsfield, Australia. From 2015 to 2017, he was an industrial-sponsored Post-Doctoral Research Fellow with the Global Big Data Technologies Centre, University of Technology Sydney, where he is currently a Lecturer. His research interest is in the area of base station antennas, reconfigurable antennas, phase shifters, and fibres for wireless communication and sensing. Dr. Ding is a recipient of the Australia Research Council Distinguish Early Career Researcher Award Fellow in 2020.

Richard W. Ziolkowski (M'87-SM'91-F'94-LF'2020) received the B. Sc. (magna cum laude) degree (Hons.) in physics from Brown University, Providence, RI, USA, in 1974; the M.S. and Ph.D. degrees in physics from the University of Illinois at Urbana-Champaign, Urbana, IL, USA, in 1975 and 1980, respectively; and an Honorary Doctorate degree from the Technical University of Denmark, Kongens Lyngby, Denmark in 2012.

Prof. Ziolkowski is the recipient of the 2019 IEEE Electromagnetics Award (IEEE Technical Field Award). He is a Fellow of the Optical Society of America (OSA, 2006) and the American Physical Society (APS, 2016). He served as the President of the IEEE Antennas and Propagation Society in 2005. He is also actively involved with the URSI, OSA and SPIE professional societies. He was the Australian DSTO Fulbright Distinguished Chair in Advanced Science and Technology from 2014-2015. He was a 2014 Thomas-Reuters Highly Cited Researcher.

He is currently a Distinguished Professor in the Global Big Data Technologies Centre in the Faculty of Engineering and Information Technologies (FEIT) at the University of Technology Sydney, Ultimo NSW, Australia. He became a Professor Emeritus at the University of Arizona in 2018, where he was a Litton Industries John M. Leonis Distinguished Professor in the Department of Electrical and Computer Engineering in the College of Engineering and was also a Professor in the College of Optical Sciences. He was the Computational Electronics and Electromagnetics Thrust Area Leader with the Engineering Research Division of the Lawrence Livermore National
Laboratory before joining The University of Arizona, Tucson, AZ, USA, in 1990. His current research interests include the application of new mathematical and numerical methods to linear and nonlinear problems dealing with the interaction of electromagnetic and acoustic waves with complex linear and nonlinear media, as well as metamaterials, metamaterialinspired structures, nano-structures, and other classical and quantum applications-specific configurations

Y. Jay Guo (Fellow'2014) (Fellow, IEEE) received the Bachelor and Master degrees from Xidian University, Xi' an, China, in 1982 and 1984, respectively, and the Ph.D. degree from Xian Jiaotong University, Xi'an, China, in 1987.

His research interests include antennas, mm-wave and $\mathrm{THz}$ communications and sensing systems as well as big data technologies. He has published over 470 research articles including 250 journal articles, most of which are in IEEE TRANSACTIONS, and he holds 26 patents.

Dr. Guo is a Fellow of the Australian Academy of Engineering and Technology, a Fellow of IEEE, and a Fellow of IET, and was a member of the College of Experts of Australian Research Council (ARC, 2016-2018). He has won a number of most prestigious Australian Engineering Excellence Awards (2007, 2012) and CSIRO Chairman's Medal (2007, 2012), and was named one of the most influential engineers in Australia in 2014 and 2015, respectively. He is a Distinguished Professor and the Director of Global Big Data Technologies Center (GBDTC) at the University of Technology Sydney (UTS), Sydney, Australia. Prior to this appointment in 2014, he served as a Director in CSIRO for over nine years. Before joining CSIRO, he held various senior technology leadership positions in Fujitsu, Siemens, and NEC in the U.K. He has chaired numerous international conferences and served as guest editors for a number of IEEE publications. He is the Chair of International Steering Committee, International Symposium on Antennas and Propagation (ISAP). He was the International Advisory Committee Chair of IEEE VTC2017, General Chair of ISAP2022, ISAP2015, iWAT2014 and WPMC'2014, and TPC Chair of 2010 IEEE WCNC, and 2012 and 2007 IEEE ISCIT. He served as Guest Editor of special issues on "Antennas for Satellite Communications" and "Antennas and Propagation Aspects of 60-90 GHz Wireless Communications," both in IEEE TRANSACTIONS ON ANTENNAS AND PROPAGATION, Special Issue on "Communications Challenges and Dynamics for Unmanned Autonomous Vehicles," IEEE JOURNAL ON SELECTED AREAS IN COMMUNICATIONS (JSAC), and Special Issue on "5G for Mission Critical Machine Communications," IEEE Network Magazine. 